Word-Association Task for Drug Use (WAT-DUD)*: A new implicit task in drug use disorder Pedro J. Pérez Moreno, Fermín Fernández Calderón, Pilar Cáceres Pachón, Javier Romero Guzmán and Jesús Gómez Bujedo.

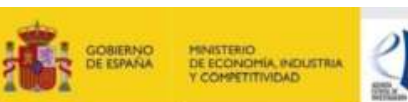 \\ de Econom supported by the Ministerio (Spain)} under Grant "PSI2016-79368-R”.

\section{1}

Introduction

In the field of addiction research, a usual aim is to predict the outcome of a treatment. Interpretation bias tasks, and especially word association tasks, have shown a moderate relationship with the use of substances.

But these studies have at least two limitations.

1) Most word association studies have been done with non-clinical samples.

2) Word association tasks require the categorization of the responses by independent raters, making them difficult to evaluate.

To solve this problems, the WATDUD was designed as a conditional discrimination, where an image (explicit or ambiguous) worked as sample, and two words (one drugrelated and another non-drugrelated) worked as comparisons.

\section{Objective:}

To provide evidence of reliability and validity for the WAT-DUD in a clinical sample

\section{Method}

Participants

The task was completed by 186 patients (90\% men, 67 outpatients, 119 inpatients).

\section{Measures}

Substance Dependence Severity Scale Cocaine Craving Questionnaire-Now

Multidimensional Craving Scale

\section{WAT-DUD: Type of trials}

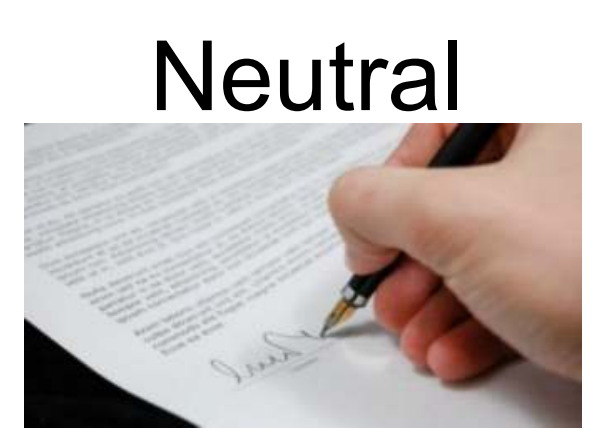

To ensure stimulus control by semantic relations (Correct in red)

Agreement - Trousers

\section{Explicit}

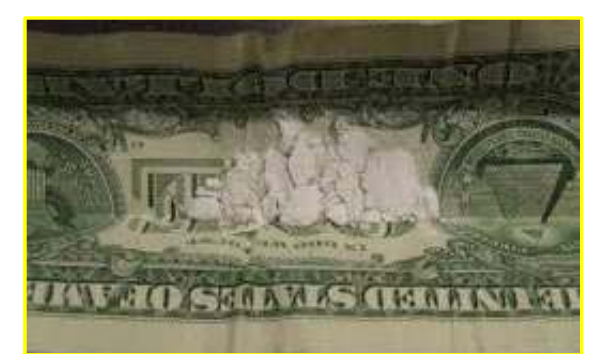

To measure possible avoidance responses

Cocaine - Shovel (Drug-related in red)

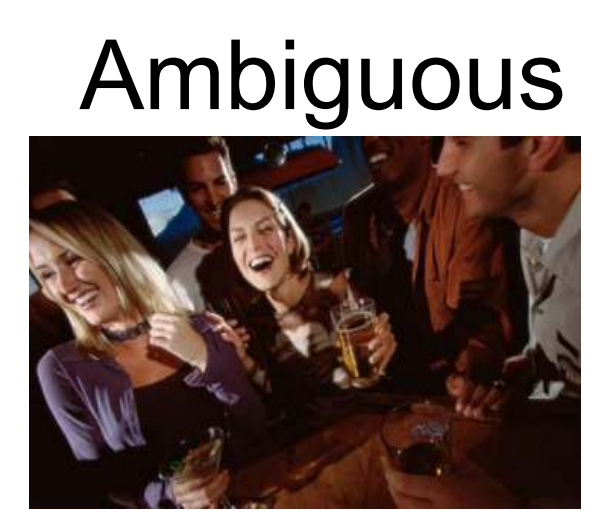

To measure interpretation bias (Drug-related in red)

Alcohol - Friends

100 trials: 25 Control, 25 Explicit, 50 Ambiguous

\title{
3 Results
}

\begin{tabular}{|c|c|c|}
\hline & Effect size & p- value \\
\hline \multicolumn{3}{|l|}{ Evidences of Reliability } \\
\hline Internal consistency & $\alpha=0.80$ & \\
\hline Test-retest & $r=0.75$ & \\
\hline \multicolumn{3}{|l|}{ Evidences of Validity } \\
\hline \multicolumn{3}{|l|}{ Relation with variables of addiction } \\
\hline $\begin{array}{l}\rightarrow \text { Higher interpretation bias in users of two or } \\
\text { more substances }\end{array}$ & $d=0.38$ & .034 \\
\hline$\rightarrow$ Cocaine craving & $r=.20$ & .029 \\
\hline$\rightarrow$ Alcohol craving & $r=.30$ & .010 \\
\hline$\rightarrow$ Alcohol abstinence & $r=.31$ & .010 \\
\hline$\rightarrow$ Severity of dependence & $r=.23$ & .040 \\
\hline \multicolumn{3}{|l|}{ Relation with therapeutic process } \\
\hline $\begin{array}{l}\rightarrow \text { Fewer drug-related words among those who } \\
\text { relapsed in explicit images }\end{array}$ & $r=.38 \nabla_{\zeta}$ & $<.000$ \\
\hline $\begin{array}{l}\rightarrow \text { Shorter reaction times to drug-related words } \\
\text { among those who relapsed in explicit images }\end{array}$ & $r=.58$ & 8.000 \\
\hline
\end{tabular}

4 $\rightarrow$ D addiction processes such as interpretation bias are related to substance use.

$\rightarrow$ Craving, abstinence and severity of dependence were related to more interpretation bias.

$\rightarrow$ The avoidance-like pattern found in patients who relapse is consistent with the results of other implicit tasks.

$\rightarrow$ The psychometric properties of the task support, in a preliminary way, its validity in research contexts.

Author Contact Details: pedro.perez@dpsi.uhu.es

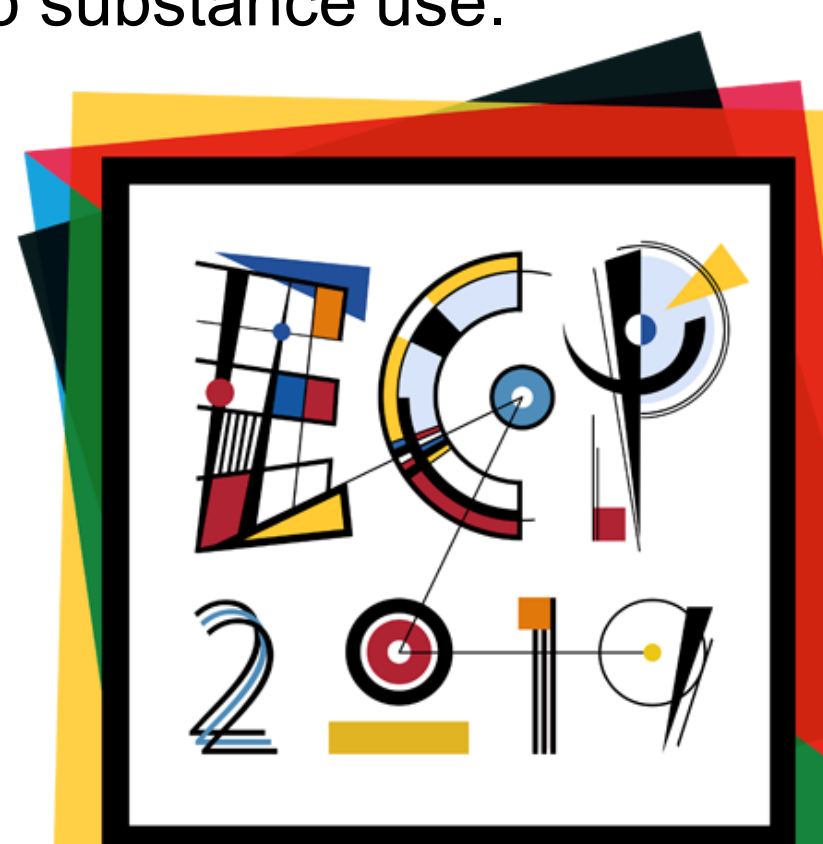

XVI European Congress of Psychology JULY 2-5 2019 MOSCOW, RUSSIA 